\title{
Charge-sign dependent modulation of cosmic ray electrons and positrons up to extreme solar maximum conditions
}

\author{
J.L. Raath*, M.S. Potgieter \\ Centre for Space Research, North-West University, Potchefstroom, South Africa \\ E-mail: janlouis.raath@gmail.com
}

\begin{abstract}
The charge-sign dependent modulation of cosmic rays is an important topic in solar modulation studies, providing insights into gradient, curvature, and current sheet drifts. Such studies, at higher levels of solar activity, have always been associated with challenges due to the limitations of numerical modulation models. We employ a three-dimensional numerical model based on stochastic differential equations and, owing to the numerical stability of this model, avoid such limitations at solar maximum conditions. We are therefore able to model results up to extreme solar maximum conditions, including the period of time over which the polarity reversal in the solar magnetic field takes place. We use our model to illustrate the effects of the tilt angle of the heliospheric current sheet that changes over the course of the solar activity cycle, and also discuss the effects when this is accompanied by a corresponding change in the magnitude of the heliospheric magnetic field. Given the results shown in this progress report, we expect our model to be able to reproduce recent data from AMS-02 and PAMELA, as well as provide us with useful predictions regarding the extent of drift effects during the next solar cycle.
\end{abstract}

35th International Cosmic Ray Conference - ICRC2017

10-20 July, 2017

Bexco, Busan, Korea

\footnotetext{
*Speaker.
} 


\section{Introduction}

When cosmic rays (CRs) enter the heliosphere, they are modulated by the turbulent solar wind (SW) and its embedded heliospheric magnetic field (HMF). This modulation is brought about by the processes of convection, particle diffusion, particle drifts, and adiabatic energy changes. Particles of different charge sign are discriminately modulated by drifts, which occur due to the gradient and curvature of the HMF as well as the heliospheric current sheet (HCS); this modulation is therefore charge-sign dependent. During an $A>0(A<0)$ polarity cycle, where the solar magnetic field is pointed outwards (inwards) in the northern heliohemisphere, positively (negatively) charged particles drift inwards mainly over the polar regions of the Sun (via the HCS in the equatorial region of the Sun) and out along the HCS in the equatorial regions (over the polar regions of the Sun). This gives rise to a 22-yr cyclic variation in CR intensities, related to the polarity of the HMF and the waviness of the HCS; this was first predicted and illustrated by [4]. See [16] for detailed illustrations of how CR particles actually drift and propagate in the heliosphere. The study of such charge-sign dependent modulation is the most effective in determining the extent of drifts during the modulation process and these studies are, as such, important; see e.g. [3, 9, 10, 11, 18].

With high precision data becoming available from satellites such as PAMELA and AMS02 , e.g. $[1,2]$, numerical modulation models can also be utilised with increasing accuracy to explain these observations. The most extensively utilised type of numerical modulation model which is employed in solar modulation studies, is that based on the finite difference approach of the alternating direction implicit (ADI) method (e.g. [6, 8]). These models, however, are known to become unstable when solving equations in higher dimensions; this can severely restrict application of these models. We use a different type of numerical model: one based on the solution of an appropriate set of stochastic differential equations (SDEs). This model has its roots in that of $[15,16]$ and has been applied to various aspects of solar modulation recently by [12, 13]; see also the detailed discussion of the mathematics and practical implementations of SDEs by [5].

In this paper, an SDE-based model is used to investigate charge-sign dependent modulation over the course of the solar activity cycle, up to extreme solar maximum conditions. This is done by looking at positron to electron ratios $e^{+} / e^{-}$over consecutive solar cycles. We also investigate the effect of changes in the drift and diffusion coefficients brought about by a changing HMF magnitude. We conclude that, given the ease with which our model is able to model situations up to the highest levels of solar activity, we should in future be able to reproduce the data that is available, $[1,2]$. The model should also be able to make valuable predictions regarding the extent of drift effects to be expected during the next solar cycle.

\section{The numerical model}

The model is based on solving the well-known Parker transport equation [7], which includes all of the relevant transport mechanisms pointed out above. These processes modulate the local interstellar spectra (LISs) of cosmic rays throughout the heliosphere so that a solution is calculated at Earth. The LISs are specified at the heliopause (HP) at a radial distance of $120 \mathrm{AU}$, which constitutes the outer modulation boundary. The LISs for positrons and electrons are those used by [17]. We use a heliospheric magnetic field (HMF) that is modified according to the modification of 
[14]. The SW profile is dependent on both latitude and solar activity, forming regions of fast SW over the polar regions and slow SW in the equatorial regions.

For the purposes of this work, it is important to point out that both the drift and diffusion coefficients employed in our model follow a $1 / B$ dependence, i.e. it is inversely proportional to the magnitude of the HMF. The features and properties of the model are discussed in detail by $[12,13]$.

\section{Results}

Drift effects can be studied by means of CR intensity as a function of the HCS tilt angle, $\alpha$, where $\alpha$ is used as a proxy for solar activity. Figure 1 shows such a profile for $750 \mathrm{MV}$ positrons at Earth during both an $A>0$ (upper blue line) and an $A<0$ (lower blue line) polarity cycle; the zero drift solution (green line) is also included and is denoted by $A=0$. Note that the zero-drift solution is not entirely independent of $\alpha$, since the SW profile is dependent on solar activity through changing $\alpha$. In these solutions, only $\alpha$ is changed over the progression of the solar cycle - all other quantities, including $B$, remains fixed.

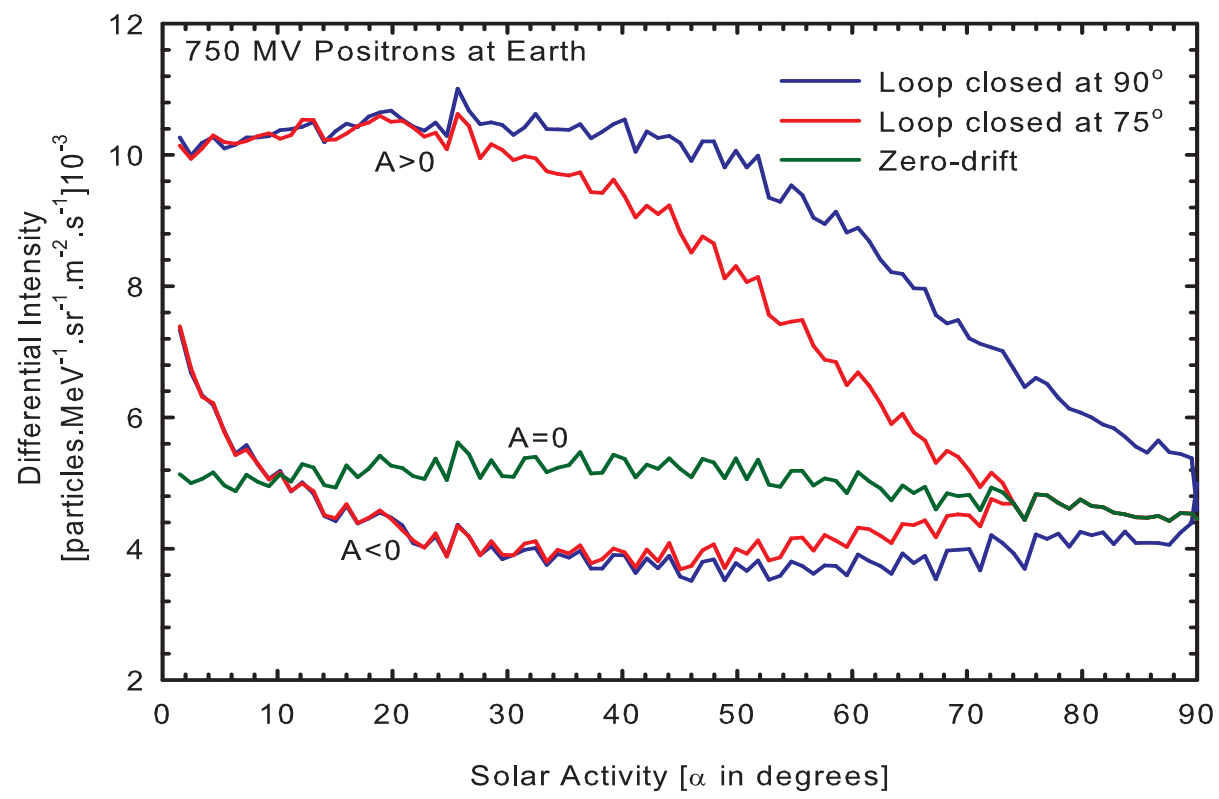

Figure 1: Computed intensities of $750 \mathrm{MV}$ positrons at Earth as a function of solar activity, proxied by $\alpha$. The upper and lower blue lines show the $A>0$ and $A<0$ solutions respectively, closing up at a theoretical maximum of $\alpha=90^{\circ}$. The red lines show the solutions where the loop closes at the maximum observational value, i.e. at $\alpha=75^{\circ}$. The green line shows the zero-drift solution $A=0$. In these solutions, the tilt angle changes linearly from $0^{\circ}$ to $90^{\circ}$.

The $A>0$ solution initially increases somewhat, due to the effect of the $\alpha$ dependent SW profile. Except for this slight increase, the $A>0$ solution initially stays more or less flat, i.e. the intensities of positrons in the $A>0$ polarity cycle are initially unresponsive to changes in the tilt angle. This is because positrons drift in over the polar regions during this cycle and changes in the 
waviness of the HCS, which occupy the equatorial regions when $\alpha$ is small, are thus not immediately reflected in the intensities. Only at $\alpha \approx 40^{\circ}$ does the $A>0$ solution start to decrease as the expanding HCS now enters higher latitudinal regions. Consequently a gradual drop in intensities is evident. Over the last few degrees the intensity drops sharply as the HCS enters the regions very close to the poles; at this stage, the drift pattern is wiped out so that intensities have to decrease even further toward those of the $A=0$ solution. At the theoretical maximum value $\alpha=90^{\circ}$, the $A>0$ solution joins with the zero-drift solution.

The $A<0$ solution shows an immediate reaction to changes in $\alpha$, since positrons now drift in via the HCS in the equatorial regions. As the waviness of the HCS expands, less positrons reaches the Earth because they have a longer path to travel along the HCS and thus have a larger probability to be scattered from the HCS by diffusion, never to reach the Earth. Intensities therefore initially drop relatively fast until it flattens off at $\alpha \approx 45^{\circ}$. It then stays flat up to $\alpha \approx 70^{\circ}$, where it starts increasing again when the HCS enters latitudes closer to the poles; at the largest tilt angles just before $90^{\circ}$ there is a sharp increase as the HCS is now very close to the poles and obstructs the drift pattern. At $\alpha=90^{\circ}$, the $A<0$ solution also ends at the levels of intensity of the zero-drift solution to join up with the $A>0$ solution to form a closed loop.

This closing up of the loop between the solutions for the $A>0$ and $A<0$ polarity cycles that occurs at the theoretical maximum of $\alpha=90^{\circ}$ is a property of our model $[12,13]$. In this work, we close the loop at a lower value of $\alpha=75^{\circ}$, since calculations based on observations never show values higher than this (see Figure 4). This is done by multiplying the drift coefficient with a function such that the properties of both the $A>0$ and $A<0$ solutions discussed above are retained, i.e. the new solutions progress in the same qualitative way as a function of $\alpha$ and both still meet the zero-drift solution to form a closed loop between the $A>0$ and $A<0$ solutions, but this time at $\alpha=75^{\circ}$, which is more realistic. These results are also shown in Figure 1 (red lines).

More insight is gained into drift patterns by plotting the intensities of Figure 1 over consecutive solar cycles. This is done in Figure 2, where the red line shows $750 \mathrm{MV}$ positrons starting at the solar maximum $\alpha=75^{\circ}$ of an $A<0$ polarity cycle, through the associated solar minimum $\alpha=0^{\circ}$, and up to solar maximum again where it enters the $A>0$ polarity cycle; the solution then goes through the solar minimum of the $A>0$ solution and back again up to the following solar maximum. Note the sharp peak in intensities around solar minimum during the $A<0$ cycle, while it forms a plato during the $A>0$ polarity cycle. This is a consequence of the drift considerations explained when we discussed Figure 1. The intensities of the plato are higher than that of the sharp peak, since intensities are higher in the phase where the particles come drifting in over the polar regions. Also shown in Figure 2 are the intensities of $750 \mathrm{MV}$ electrons and these show a plato during $A<0$ polarity cycles, while it shows the sharp peak during the $A>0$ cycle. This is as expected, considering that that drift directions are reversed for oppositely charged particles.

An even clearer indication of charge-sign dependent modulation can be obtained by studying the $e^{+} / e^{-}$ratio. Figure 3 shows the $e^{+} / e^{-}$ratios calculated directly from the solutions of Figure 2 and we obtain the well-known "W" and " $\mathrm{M}$ " shapes during the $A<0$ and $A>0$ polarity cycles respectively. Though these results also follow directly from the drift picture that was described in the discussion for Figure 1, we can come to some additional insights by explaining this figure in more detail. Starting at the peak in the $e^{+} / e^{-}$ratio at the solar minimum $\alpha=0^{\circ}$ during the $A<0$ polarity cycle, we see that the ratio rapidly decreases as the tilt angle starts increasing because 


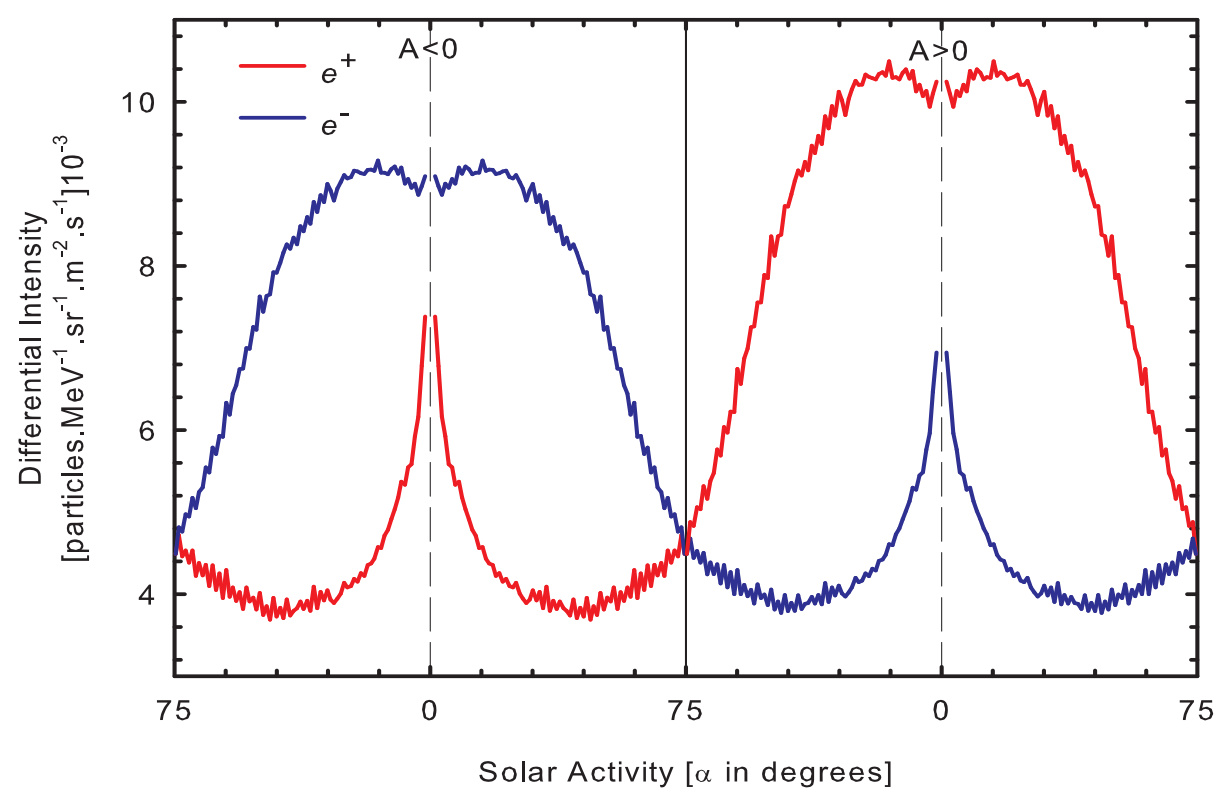

Figure 2: Differential intensities of $750 \mathrm{MV}$ positrons (red lines) and electrons (blue lines) as a function of solar activity, proxied by the tilt angle $\alpha$. Solar minimum is indicated by the vertical long-dashed lines and solar maximum by the vertical solid line. The tilt angle changes linearly from an $A<0$ to an $A>0$ cycle.

positron intensities, with positrons drifting inward along the HCS, will immediately decrease with an increase in the waviness of the HCS, while electron intensities will remain largely unaffected. The $e^{+} / e^{-}$ratio then flattens off when any increase in the HCS waviness is no longer effective in reducing positron intensities. When the HCS enters the higher latitudinal regions, it starts affecting electron intensities, i.e. electron intensities now start decreasing, while positron intensities remain unaffected. This causes the $e^{+} / e^{-}$ratio to increase and keep on doing so up to the maximum $\alpha=75^{\circ}$. Note that the ratio, at this point, is higher than at solar minimum conditions around $\alpha=0^{\circ}$. When we look at the ratio during the $A>0$ polarity cycle, we can construct a similar explanation by following the exact same reasoning.

Next, values of $\alpha$ that were calculated based on observations are used; these values are calculated for each Carrington rotation and are shown in Figure 4. The values of all other parameters, including the HMF magnitude $B$ are still kept constant. The solutions for these values of $\alpha$ are shown by the red scatter plot in Figure 3. As expected, the solutions for these tilt angles are centered around the black lines; all differences between the red scatter plot and the black line can be ascribed to stochastical variation.

The green scatter plot in Figure 3 shows solutions that were calculated for the tilt angles shown in Figure 4, but where each value of $\alpha$ was now also accompanied by a corresponding change in $B$, observed at the Earth, which changes the diffusion and drift coefficients directly; these values of $B$ have been averaged over 27 days, i.e. $\sim$ one Carrington rotation, and are also depicted in Figure 4. Note that this input occurs over the so-called transition region, i.e. the period of extreme 


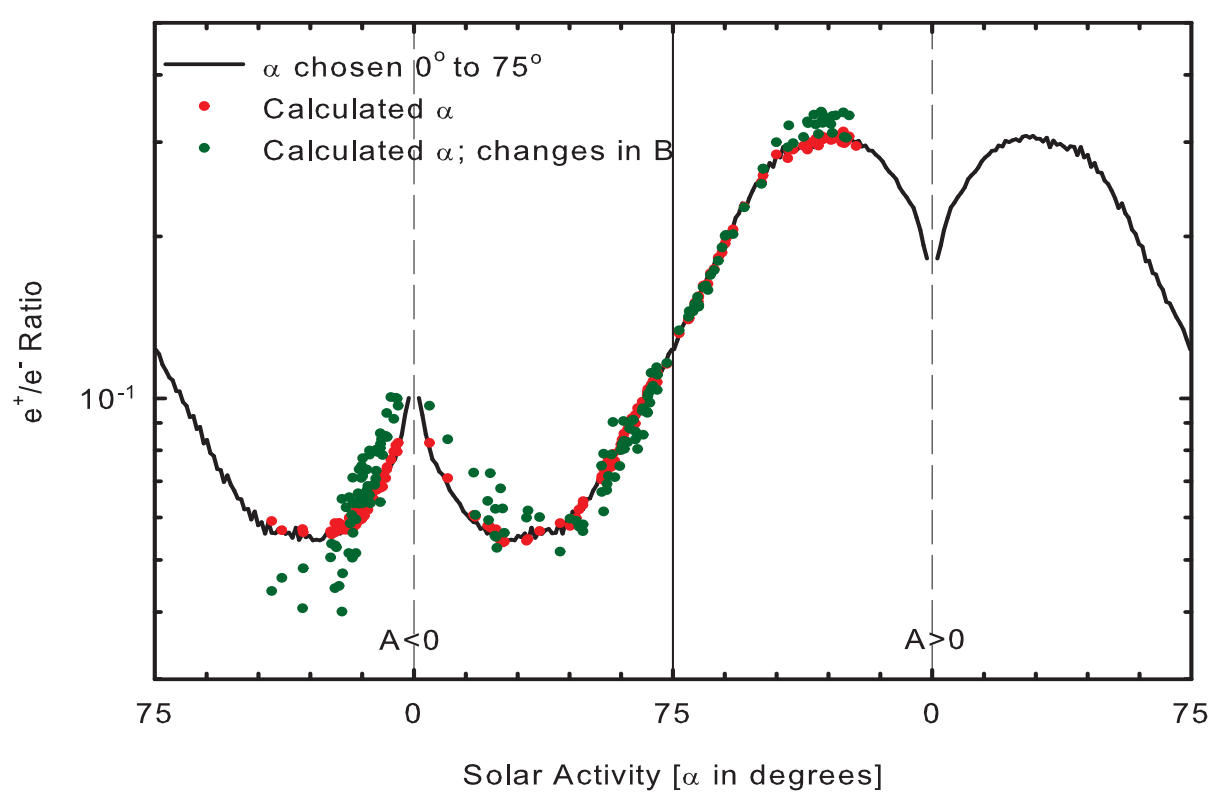

Figure 3: This figure shows the $e^{+} / e^{-}$ratios for three cases. The first case (black lines) shows the ratios where the values of $\alpha$ were chosen to range linearly from $0^{\circ}$ to $75^{\circ}$ over consecutive solar cycles, from an $A<0$ to an $A>0$ cycle; the second case (red scatter plot), shows the ratios where we have used the calculated tilt angles from Figure 4; the third case (green scatter plot), shows the solution when using the calculated $\alpha$, but also included corresponding changes in $B$. Note that clustering in the scatter plots corresponds to a flattening in the values of $\alpha$ or $B$ in Figure 4 .

solar maximum conditions, where no clear HMF polarity is defined. The differences between the green and red scatter plots are significant, and more so around solar minimum condition. During most of solar maximum the differences are smaller. Starting at the solar maximum in the middle of the $A<0$ and $A>0$ solar minima, the differences become most significant during and after the flattening that occurs in the $e^{+} / e^{-}$ratio. The difference is most significant during the flattening left of the first solar minimum when $B$ changed most, and reaches a decrease in the green solution of a factor 0.69 relative to the red solution. Just after this solar minimum, the green solution is above the red solution. Evidently, despite these deviations, the trend set by the black line is followed.

To account for the detail of these differences requires some further research, but the following must be considered for any such explanation. When a change in $B$ is introduced, it enters into both the drift and diffusion coefficients through its $1 / B$ dependence. It is now important to keep in mind that drift effects are affected in two ways: first, through the drift coefficient with its $1 / B$ dependence and, second, through the change in intensity gradients that result from any change in the diffusion coefficient, which also follows $1 / B$. Any changes in the drift effect due to a change in $B$ will thus have to be explained in the light of this argument; see also [11].

Our aim with this model is to eventually explain in detail the observations from PAMELA and AMS-02 [1, 2]. To do this, we will have to look at the way in which the drift and diffusion 


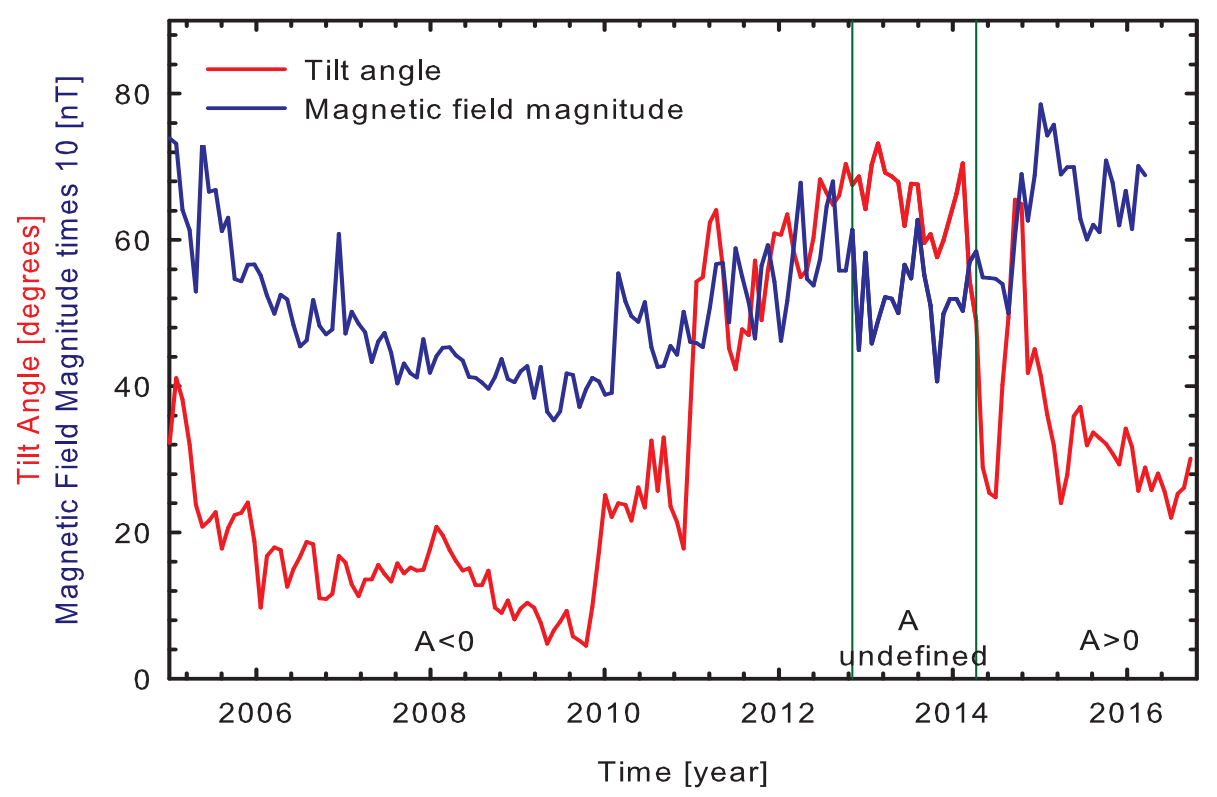

Figure 4: This figure shows the values of the tilt angles from http://wso.stanford.edu as well as the 27 day averaged values for the magnetic field, obtained from http://cohoweb.gsfc.nasa.gov, that we have used in Figure 3. Note that the region between the two vertical green lines is the so called transition region, i.e. the region where there is no clearly defined solar magnetic field polarity.

coefficients scale with $B$, especially during extreme solar maximum conditions and how much drifts remain effective during this period. The simple $1 / B$ approach probably will not be sufficient and we will have to look at the various options available when it comes to this (see e.g. [6]). However, given the numerical stability of our model and the ease with which we can produce results even at extreme solar maximum conditions, it should be an obtainable goal. We should also be able to make valuable predictions regarding the extent of drift effects during the next solar cycle.

\section{Conclusions}

Our model is able to describe charge-sign dependent modulation up to the highest levels of solar activity, with characteristic features for positrons and electrons. It is also illustrated how modulation is influenced by changes in the drift and diffusion coefficients brought about through its $1 / B$ dependence. Given the numerical ease with which the model handles these changes, especially during extreme solar maximum conditions, we are positive that with further refinements to the drift and diffusion coefficients it should be possible to reproduce high precision observations $[1,2]$. We will also be able to make predictions regarding drift effects during the next solar cycle.

We acknowledge the partial financial support of the South African National Research Foundation (NRF) as well as the South African National Space Agency (SANSA). J.L. Raath also thanks R.D. Strauss for discussions regarding SDE modelling. 


\section{References}

[1] Adriani O., Barbarino G. C., Bazilevskaya G. A., et al., (PAMELA collaboration), Time dependence of the electron and positron components of the cosmic radiation measured by the PAMELA experiment between July 2006 and December 2015. Phys. Rev. Letters, 116, 241105 (2016)

[2] Aguilar, M., Aisa, D., Alvino, A., et al., (AMS Collaboration), Electron and poitron fluxes in primary cosmic rays measured with the alpha magnetic spectrometer on the international space station, Phys. Rev. Lett., 113, 121102 (2014)

[3] Di Felice, V., Munini, R., Vos, E.E., Potgieter, M.S., New evidence for charge-sign dependent modulation during the solar minimum of 2006 to 2009, ApJ., 834, 89-97 (2017)

[4] Jokipii, J.R., Thomas, B., Effects of drift on the transport of cosmic rays. IV - Modulation by a wavy interplanetary current sheet, ApJ., 243, 1115-1122 (1981)

[5] Kopp, A., Büsching, I., Strauss, R.D., Potgieter, M.S., A stochastic differential equation code for multidimensional Fokker-Planck type problems, Comput. Phys. Commun., 183, 530-542 (2012)

[6] Ngobeni, M.D., Potgieter, M.S., Modelling the effects of scattering parameters on particle-drift in the solar modulation of galactic cosmic rays, Adv. Space Res., 56, 1525-1537 (2015)

[7] Parker, E.N., The passage of energetic charged particles through interplanetary space, Planet. Space. Sci. 13, 9-49 (1965)

[8] Potgieter M. S., The heliospheric modulation of galactic electrons: consequences of new calculations for the mean free path of electrons between $1 \mathrm{MeV}$ and $10 \mathrm{GeV}$., J. Geophys. Res., 101, A11, 24411-24422 (1996)

[9] Potgieter, M.S., Moraal, H., A drift model for the modulation of galactic cosmic rays, ApJ. 294, 425-490 (1985)

[10] Potgieter, M.S., Langner, U.W., Heliospheric modulation of cosmic-ray positrons and electrons: effects of the heliosheath and the solar wind termination shock, ApJ., 602, 993-1001 (2004)

[11] Potgieter, M.S., Vos, E.E., Difference in the heliospheric modulation of cosmic-ray protons and electrons during the solar minimum period of 2006 to 2009, Astron. Astrophys., 601, A23, 1-10 (2017)

[12] Raath, J.L., Strauss, R.D., Potgieter, M.S., New insights from modeling the neutral heliospheric current sheet, Astrophys. Space Sci., Astropys. Space Sci., 360, 24, 1-13 (2015)

[13] Raath, J.L., Potgieter, M.S., Strauss, R.D., The effects of magnetic field modifications on the solar modulation of cosmic rays with a SDE-based model, Adv. Space Res., 57, 9, 1965-1977 (2016)

[14] Smith, C.W., Bieber, J.W., Solar cycle variation of the interplanetary magnetic field spiral, ApJ., 370, 435-441 (1991)

[15] Strauss, R.D., Potgieter, M.S., Büsching, I., Kopp, A., Modeling the modulation of galactic and Jovian electrons by stochastic processes, ApJ., 735(83), 1-13 (2011)

[16] Strauss, R.D., Potgieter, M.S., Büsching, I., Kopp, A., Modeling heliospheric current sheet drift in stochastic cosmic ray transport models, Astrophys. Space Sci., 339, 223-236 (2012)

[17] Vos, E.E., Modelling charge-sign dependent modulation of cosmic rays in the heliosphere, Ph.D. dissertation, North-West University (2016)

[18] Webber, W.R., Potgieter, M.S., Burger, R.A., A comparison of predictions of a wavy neutral sheet drift model with cosmic-ray data over a shole modulation cycle: 1976-1987, ApJ. 349, 634-640 (1990) 